\title{
Oral English Testing of English-Majors From the Perspective of Communicative Language Testing Theory

\author{
Xiao Jing ${ }^{1, a^{*}}$, Wang Jing ${ }^{2, b}$
}

${ }^{1.2}$ Foreign Languages Department of Zhuhai College Jilin University, Zhuhai, Guangdong, China axjangel@126.com, bwangjing_0013@163.com

Keywords: Communicative language testing theory, English-majors, oral testing

\begin{abstract}
English testing plays an important role in language learning and teaching, serving as a guidance for teachers and students. A scientific and effective oral test will bring positive backwash effect on college English oral teaching. Different from the traditional language testing, communicative language testing emphasizes the social function of a language, and has been a heated topic and popular trend in language testing. Based on communicative language testing theory, this paper analyzes the present situation and main problems in the oral English Testing system for college students and explores the application of communicative language testing theory on college oral English testing for English-majors
\end{abstract}

\section{交际语言测试理论视野下的英语专业口语测试}

\author{
肖婧 $1, a^{*}$ ，王晶 $2, b$ \\ 1,2吉林大学珠海学院外语系, 珠海, 广东, 中国 \\ axjangel@126.com, bwangjing_0013@163.com
}

关键词: 交际语言测试理论，英语专业，口语测试

中文摘要. 测试是语言教学与学习过程中的重要环节, 是教与学的导向。科学合理的口语测 试能为口语教学提供积极的反馈, 是提高教学质量及调整学生学习策略的有效途径。交际语 言测试是有别于传统语言测试，交际语言测试理论强调语言的社会功能，交际语言测试理论 强调语言的社会功能, 是当前语言测试研究的一个热门课题。从交际语言测试的理论出发, 对我国英语专业口语测试现状及问题进行了分析, 探讨了交际语言测试在大学英语专业口语 测试中的应用。

\section{1. 引言}

《高等学校英语专业教学大纲》(2000)明确规定, 在专业英语教学中要注重培养学生的跨 文化交际能力; 并详细地规定了学生在口头表达方面应达到的要求。随着高校英语教学改革 的深入开展, 学生的语言交际能力受到了更多的关注。测试与教学相辅相成, 口语测试是口 语教学过程中的重要环节, 它可以有效地考核学生实际运用语言的能力; 同时, 科学、合理、 有效的口语测试能为教学提供积极的反馈, 是提高专业口语教学质量的必要保证。

\section{2. 交际语言测试理论的渊源}

二十世纪九十年代, 美国著名应用语言学家Bachman在语言学家Canale和Swain的研究基 础上, 提出了交际语言能力模型。他的观点受到了国际语言测试界广泛关注, 也成为了交际 
语言测试的理论基础。他认为交际是语言和环境动态交换的过程, 交际语言的使用不是简单 地产生或解释话语, 而是以话语及其发生的环境的关系为特征的。他将语言交际能力分为三 个部分: 语言能力、策略能力和心理-生理机制。语言能力包括语言组织能力和语言使用能力; 策略能力是指为实现某个交际目的而选择最有效方法的能力; 心理生理机制本质上指语言使 用的实施阶段所牵涉的心理和生理过程, 如在接收语言过程中使用的是视听技能, 而在产生 语言过程中使用的是神经肌肉技能[1]。交际语言测试就是根据这一理论孕育而生了。语言测 试经历了三代不同的体系:科学前测试体系、结构主义测试体系和交际测试体系。交际测试属 于第三代测试体系, 它不仅仅测试语言知识, 而是把测试的重点放在运用语言的能力上, 即 着重了解受测者用目标语进行交际的能力。交际语言测试理论的把语言的运用看成是一个动 态的过程, 重视语言在具体环境及社会文化背景下的使用, 强调语言能力不仅仅指学习者对 语言知识的掌握, 更重要的是运用所掌握的知识进行有效交际。

\section{3. 我国英语专业口语测试的现状及问题}

长期以来，传统的英语专业教学模式以培养学生的语言能力作为重心，讲授语音、语法、 词汇等系统语言知识, 忽视了学生的语用能力。在许多高校的专业课授课中, 教师的授课只 停留在语言形式的层面上, 要求学生反复掌握语法规则, 把语言视为一种静态的事物, 没有 让学生理解语言本质; 这就造成了许多学生虽然掌握大量语言知识, 但学生的语言交际能力 却未能得到有效的提高。在我国, 外语领域的口语测试也只是在最近十几年随着经济的发展 和对外交流的增多才被提到英语教学的议事日程上来; 起步较晚。英语专业的四级口试1994 年试点, 1999年正式向全国推行; 而专业八级口语测试2001年才开始实行; 或许是由于起步晚, 我国英语专业的口语测试中存在许多的问题[2]。

随着高校英语教学改革的深入开展, 各个大学开始纷纷重视口语课程, 并陆续在英语测 试中增添了口语测试的环节; 教育部有明确的外语专业教学大纲, 其中对外语专业口语测试 也出了具体要求。但实际上外语专业口语测试的实施主体是高校的外语系, 在日常的英语专 业口语课程中, 口语测试模式五花八门, 没形成统一的模式, 也无统一的标准。因此许多教 师往往参考大学英语口语测试的模式, 采用直接型口试: 由教师提供信息, 教师和学生就某 一话题进行一对一交谈, 交谈时采用教师提问学生回答的形式; 有时还考查学生就所抽定的 题目 (图片或文字) 作简短描述、阐述个人观点等, 总的来看还是侧重于对语音语调是否正 确, 语法用词是否正确以及在测试中能否流利地回答问题三个方面的考核。但是, 这种模式 无论从测试的内容还是从评估的方式来看, 没有按照交际口语测试的设计原则、形式、没有 严谨的评分方法及评分标准, 也就谈不上口语测试的效度和信度。学生只要准备好规定方面 的内容就可以了, 这种测试方式在真实性、交际性和互动性方面都有一定的缺陷; 它无法从 不同的侧面、不同的层次测试学生的口语交际能力, 对教学起不到有效的反拨作用。

\section{4. 交际语言测试理论在英语专业口语测试中的应用原则}

\section{1 口语测试的题型不应单一, 且应考虑学生的程度}

口语测试与其它类型的英语测试相比, 如语法测试、阅读能力测试或听力测试更具有主 观性; 直接影响了口语测试的信度和效度。由于口语测试中评估的主观性决定了口语测试的 题型不应单一，只有选择多种题型，才能从不同侧面、不同层次反映学生真实的口语水平， 同时也能提高测试的信度和效度。在试题的设计上应根据学生的实际听说能力, 考虑到测试 者与被测试者之间存在信息交流与互动,话题应具有一定的广泛性和代表性。教师应该仔细而 全面地分析学生现在或将来需要使用英语的场合, 选择典型情景并具有覆盖面广、新颖实用 的特点来确定口语考试的内容和题型。 


\section{2 真实性原则应在口语测试的内容设计中遵循}

口语测试内容是否具有真实性, 直接关系到测试的信度和效度, 同时口语考试的任务是 把考试能力与社会交际能力联系起来的桥梁, 没有口头表达的真实性, 也就很难反映考生的 口语交际能力。在英语口语交际过程中,如果交际双方或一方违反了语言表达的贴切性和得体 性,就会犯语用错误。何自然曾指出使用语言不能只讲究遣词造句的正误。更重要的是语言使 用是否合适、得体。只有充分重视英语口语测试中的语用要求,才能确保测试的真实”。杨惠 中也对交际和交际过程的这样描述, “从交际法的角度来看, 所谓掌握一门语言是指在一定 的语境中能够使用所学的语言进行有效的交际, 交流思想感情, 达到相互沟通的目的。从语 言作为交际工具的角度来看，仅仅掌握语言形式是不能够进行有效的交际的,因为语言交际过 程 涉及到交际的目的、语境、彼此的角色地位等等; 同样的语言形式, 由不同的人在不同的 场合以不同的方式讲出来, 其含义可能完全不同, 因此语言交际过程实际上是一种解释过程

（interpretation），是交际双方的协同过程（negotiation）,既然如此，语言测试就必须在真实 的 (authentic) 语境中采用真实材料来进行, 观察学生在真实语境中运用语言达到交际目的 的能力, 并以此来判断学生的语言水平。”

因此, 口语测试的内容设计上要始终贯彻真实性原则, 形式上应该尽量模拟现实生活中 真实交际的情形, 才能推测考生在目的语使用域中的语言使用能力; 而且测试的反馈作用能 促使学生学习真实的语言材料, 从而有利于提高他们的语言交际能力。考试的内容应覆盖考 生所熟悉的一般性话题, 如学校生活、家庭生活、业余爱好, 众所周知的社会问题等, 同时 可以针对高年级学生不同的专业方向设计与专业知识相关的常识性话题, 如针对旅游英语专 业的景点介绍、商务英语专业的商务谈判过程等。与学生的生活、学习、家庭和专业相关的 话题会使考生无论口语程度好坏, 都能有话可说, 有发挥的空间, 在真实的情境里真实地表 达; 但要注意的是在口语测试的内容应避免专业性较强的话题, 因为它不能公正地考查应试 者的真实口语水平。

\section{3 测试的交互性应在口语测试中体现}

语言学家Weir(1993)认为: 体现一个人交际能力的有两个方面：一方面是对信息的组织, 另一方面是对交际互动方式的组织。学生要想尽快获得一般交际的能力, 如一般性问候、道 歉、自我介绍、寻求信息等, 就必须学习这些日常用语及其使用的话语环境。互动能力是英 语口语交际能力的另一个重要方面, 指的是在自己说过之后, 能够大致预计对方会怎样答复, 并且有能力把握谈话内容的发展。这两个方面也正是交际口语测试中需要考查学生的, 也可 以说交际口语测试不仅考查学生自身的语言产出, 也包含使用语言与他人进行交际的能力。 与传统语言测试不同, 交际测试中的考生则是 “局内人” ，他参与语言交际测试即互动的过 程, 并与交际对象合作/协商达到信息交流, 对这个过程的评价得出测试结果(武尊民, 2002) 因 此, 交际口语测试中的交互性是指考官与考生之间, 或者考生与考生之间要围绕某个话题进 行交流，双方或多方在一过程中不断地交换听或说的角色，尽量根据对方的需要提供信息。 达到交流的目的。在考试的过程中还应注重考试的情景性, 即将口试试题置于一个真实的情 景中, 明确规定考生所要扮演的角色和交际对方的角色, 考生按照要求做出与自己身份相 符的反应, 由此考察语言的得体性。

\section{4 结合综合法和分析法的口语测试的评分方法。}

Bachman 认为信度与效度是语言测试中的两个互补因素，应相对平衡。口语测试的评分 方法有综合法(holistic approach)和分析法(analytic approach)两种。基于交际语言测试理论, 英 语口语测试评分方式应该采用分析法和综合法相结合的方式。综合法是评分人员根据一定的 标准和整体印象划分等级; 综合评分法是依据交际性测试体系的观点, 把语言放在实际交际 中作为一个整体来评价, 而综合法从整体上把握考生口语表现, 效度比较高, 但信度较低, 不 
易掌握。分析法是把口试表现分解为若干要点(语音准确、语言准确、语调准确、语言流畅、 交互能力等), 评分人员根据一定的标准对不同要点分别评分, 各要点得分总和即为口试的总得 分或总级别。分析法注重口语的各组成要素, 评分方法标准统一, 信度较高, 易操作; 分析性 评分法则反映了分割式的语言观。因此, 在交际性口语测试中, 为了保证测试的质量,评估应把 整体观与分析观相统一, 既保证总体印象, 又不忽视口语的各个要素;应坚持综合评分和分析 评分相结合的原则,减小偏差,力求信度与效度的统一。同时,应采取二评方式, 即有两位考官 分别独立评分, 一名为主考官, 负责口语测试的进行和评出总体印象分; 另一名为副考官, 只负责按照评分细则，用分析法评分。考官之间不相互商议。

\section{5. 结束语}

交际语言测试是一种科学的，人性化的语言测试模式，目前它已经得到全国外语教育界 的认可; 根据这种测试理念设计的口语测试将直接影响英语专业口语教学内容和教学模式。 将它广泛应用到英语专业口语测试中, 将有效地提高英语口语教学的效率, 促进英语专业口 语教学改革。

\section{致谢}

本文为2014中国外语教育基金项目 No. ZGWYJYJJ2014A44阶段性成果之一。

\section{References}

[1] L.F.Bachman, Fundamental Considerations in Language Testing. Oxford:Oxford University Press, 1990.

[2] L.F.Bachman and A.S Palmer. Language Testing in Practice. Oxford:Oxford University Press 1996.

[3] C. J. Weir. Understanding and Developing Language Tests. NewYork: Prentice Hall 1993.

[4] C. Jie. Reflections on the reform of oral English test. Heilongjiang Researches on Higher Education .2009, vol.3. 62-63.

[5] J. Tang, L. Li, Guo Kai. Rating scales for speaking tests: What the past 30 years of development tells us. Modern Foreign Languages. 2016.vol.39.pp 853-874.

[6] Y. Huizhong. A study on the validity of College English Test Shanghai: Shanghai Foreign Language Education Press. 1998.

[7] C. A. Chapelle, M. K. Enright \& J. M. Jamieson (eds.). Building a Validity Argument for theTest of English as a Foreign Language. New York: Routledge.2008. 